04.2

\title{
Определение изотопного состава плазмы по частоте альфвеновских колебаний в токамаке ТУМАН-3М
}

\author{
() Г.И. Абдуллина, Л.Г. Аскинази, А.А. Белокуров, Н.А. Жубр, В.А. Корнев, С.В. Крикунов, С.В. Лебедев, \\ А.Д. Мельник, Д.В. Разуменко, А.И. Смирнов, А.С. Тукачинский, Ф.В. Чернышев
}

Физико-технический институт им. А.Ф. Иоффре РАН, Санкт-Петербург, Россия

E-mail: Abdullina@mail.ioffe.ru

Поступило в Редакцию 20 мая 2019г.

В окончательной редакции 20 мая 2019г.

Принято к публикации 21 мая 2019г.

\begin{abstract}
Обсуждается возможность определения эволюции изотопного состава плазмы по частоте альфвеновских колебаний в омических разрядах с импульсным напуском дейтерия в водородную плазму и водорода в дейтериевую плазму в компактном токамаке ТУМАН-3М. Полученные этим методом изотопные соотношения - относительные концентрации водорода $n_{\mathrm{H}} /\left(n_{\mathrm{H}}+n_{\mathrm{D}}\right)$ и дейтерия $n_{\mathrm{D}} /\left(n_{\mathrm{H}}+n_{\mathrm{D}}\right)$ в смешанной водороднодейтериевой плазме - качественно согласуются с результатами спектроскопических измерений и данными корпускулярной диагностики.
\end{abstract}

Ключевые слова: токамак, альфвеновские колебания, изотопный состав плазмы.

DOI: 10.21883/PJTF.2019.15.48089.17813

Осуществление самоподдерживающейся термоядерной реакции синтеза на основе слияния ядер дейтерия и трития в термоядерном реакторе будущего требует поддержания соотношения концентраций этих изотопов на заданном уровне. В связи с этим одной из основных задач, стоящих перед диагностиками токамакареактора ИТЭР, запуск которого намечен на ближайшее будущее, является контроль изотопного состава плазмы [1]. Существует несколько диагностических методик, которые предоставляют возможность измерения и контроля изотопного состава плазмы. Используя метод корпускулярной диагностики, можно определить изотопный состав исходя из соотношения потоков атомов изотопов водорода, покидающих плазму вследствие нейтрализации соответствующих ионов [2]. Для определения изотопного состава плазмы также традиционно используется спектроскопическая диагностика. C ее помощью измеряют свечение линий атомов изотопов водорода в плазме, а соотношение интенсивностей этих линий дает информацию об их относительных концентрациях на периферии плазмы [3]. Другой подход к определению изотопного состава основан на спектрометрии нейтронного излучения плазмы. Используя этот диагностический метод, который применим для случая термоядерной плазмы, работающей на смеси дейтерия и трития, информацию о соотношении этих изотопов можно получить из анализа энергетического спектра нейтронов [4,5]. Имеются также и другие, не настолько широко распространенные диагностические методы, позволяющие определять изотопное соотношение. К ним можно отнести коллективное томсоновское рассеяние, основанное на измерении спектра излучения, рассеянного на микроскопических флуктуациях электронной плотности, обусловленных движением ионов в плазме [6], а также рефлектометрию на частоте ионионного гибридного резонанса, в которой используется свойство ион-ионной гибридной отсечки изменять свое положение при изменении компонентного состава плазмы [7].

В настоящей работе рассматривается возможность определения изотопного соотношения по частоте альфвеновских колебаний (АК). АК в токамаке ТУМАН-3М наблюдаются в омическом режиме при достаточно низкой концентрации $\left(\bar{n}_{e} \leqslant 1.5 \cdot 10^{19} \mathrm{~m}^{-3}\right)$, в более плотной плазме $\left(\bar{n}_{e}>3 \cdot 10^{19} \mathrm{~m}^{-3}\right)$ АК исчезают $[8,9]$. АК распространяются в плазме с альфвеновской скоростью $v_{\mathrm{A}}=B_{\mathrm{T}} / \sqrt{\mu_{0} \sum_{i} m_{i} n_{i}}$, и для них характерно следующее дисперсионное соотношение:

$$
f_{\mathrm{A}}=k_{\|} v_{\mathrm{A}} / 2 \pi
$$

Здесь $B_{T}$ - тороидальное магнитное поле, $\mu_{0}-$ магнитная постоянная, $m_{i}$ и $n_{i}-$ масса и концентрация $i$-го сорта иона, $f_{\text {A }}$ - частота $\mathrm{AK}, k_{\|}$- параллельное волновое число. Рассмотрим плазму, состоящую из дейтерия и водорода, в которой отсутствуют примеси. В этом случае из эволюции экспериментально измеренной частоты АК $f_{\text {А }}$ можно найти сумму массовых плотностей дейтерия и водорода

$$
\sum_{i} m_{i} n_{i}=m_{\mathrm{H}} n_{\mathrm{H}}+m_{\mathrm{D}} n_{\mathrm{D}}=k_{\|}^{2} B_{T}^{2} /\left(4 \pi^{2} \mu_{0} f_{\mathrm{A}}^{2}\right) .
$$

Используя это соотношение и условие квазинейтральности плазмы $\sum_{i} n_{i} Z_{i}=n_{e}$ (электронная концентрация $n_{e}=n_{\mathrm{H}}+n_{\mathrm{D}}$ измеряется в эксперименте), можно запи- 

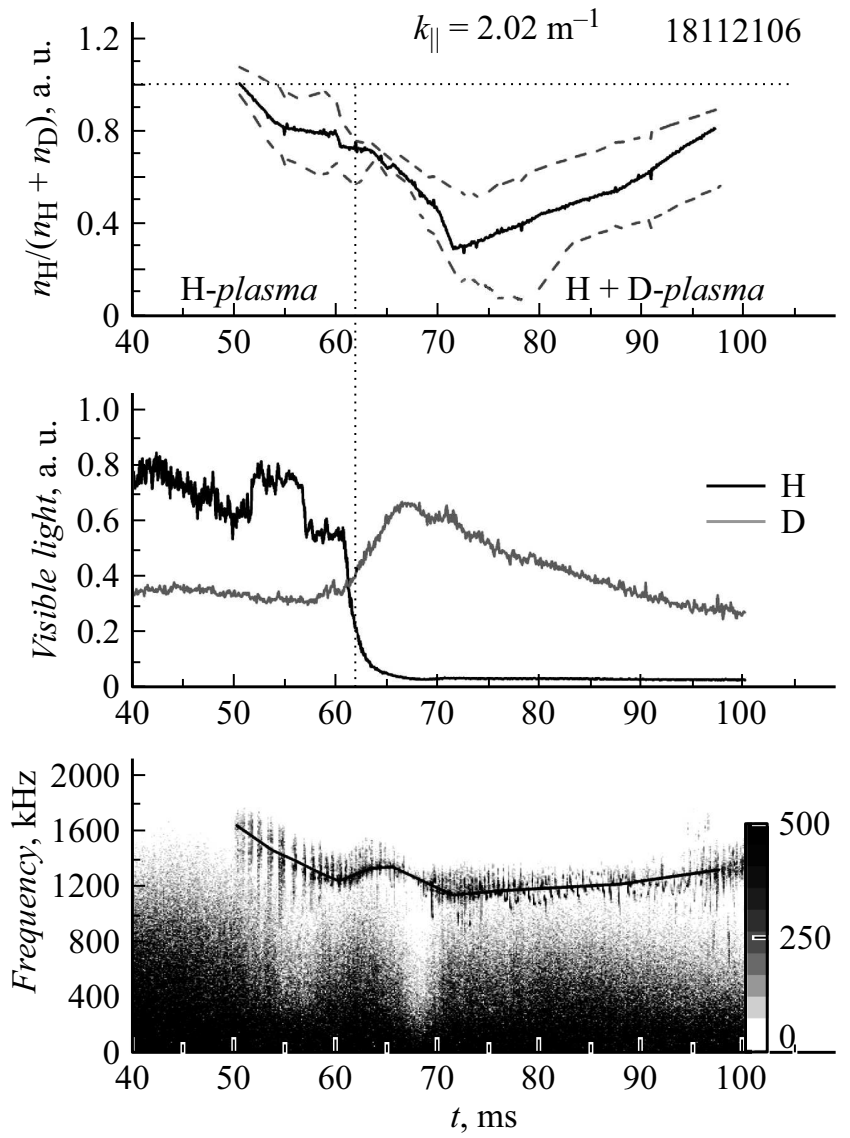

Рис. 1. Относительная концентрация водорода, излучение в видимом диапазоне из клапанов напуска газа и спектрограмма сигнала магнитного зонда в разряде 18112106.

сать выражения для концентраций водорода и дейтерия

$$
\begin{gathered}
n_{\mathrm{H}}=2 n_{e}-\sum_{i} m_{i} n_{i} / m_{\mathrm{H}}=2 n_{e}-k_{\|}^{2} B_{T}^{2} /\left(4 \pi^{2} \mu_{0} m_{\mathrm{H}} f_{\mathrm{A}}^{2}\right), \\
n_{\mathrm{D}}=\sum_{i} m_{i} n_{i} / m_{\mathrm{H}}-n_{e} .
\end{gathered}
$$

Учитывая, что АК в токамаке ТУМАН-3М локализованы в центральной части плазменного шнура [10], эти выражения можно использовать для определения изотопного соотношения в центральной части плазмы $n_{\mathrm{H}} / n_{e}(r=0 \mathrm{~cm})$ или $n_{\mathrm{D}} / n_{e}(r=0 \mathrm{~cm})$. В отличие от экспериментов на токамаке JET [11], в которых для измерения изотопного состава плазмы по спектрам АК последние возбуждались с помощью специальной антенны и генератора, в данной работе исследовались спектры АК, развивающихся в плазме „естественным“ образом.

АК в токамаке ТУМАН-3М регистрируются внутрикамерными магнитными зондами. Поскольку спектральный состав сигналов АК на всех зондах одинаков, для расчета величины изотопного соотношения использовался сигнал одного из этих зондов, установленного внутри разрядной камеры токамака, полоидальный угол $\theta=191^{\circ}$ (отсчет угла $\theta$ осуществляется от экваториальной плоскости со стороны слабого магнитного поля). Эволюция частоты АК $f_{\text {A }}$ аппроксимировалась по экспериментальным значениям частот отдельных вспышек на этом зонде (рис. 1,2). Электронная концентрация измерялась СВЧ-интерферометром с десятью вертикальными каналами, локальные значения концентрации находились из хордовых измерений посредством преобразования Абеля.

Для проверки возможности определения изотопного соотношения по частоте АК были проведены эксперименты с импульсным напуском дейтерия в водородную плазму (разряд 18112106) и водорода в дейтериевую плазму (разряд 19012819). Рассматриваемые разряды имели идентичные основные параметры плазмы: в момент времени $t=55 \mathrm{~ms}$ ток по плазме $I_{p} \approx 137 \mathrm{kA}$, магнитное поле $B_{T} \approx 0.94 \mathrm{~T}$. Сценарии напуска рабочих газов подбирались таким образом, чтобы центральные среднехордовые электронные концентрации $\bar{n}_{e} \sim 1 \cdot 10^{19} \mathrm{~m}^{-3}$ в сравниваемых разрядах отличались незначительно. Поступление газа, используемого для создания фоновой плазмы, контролировалось фотодиодом, установленным в основном клапане напуска газа и регистрирующим свечение атомов в видимом диапазоне длин волн. Поступление „примесного“ изотопа
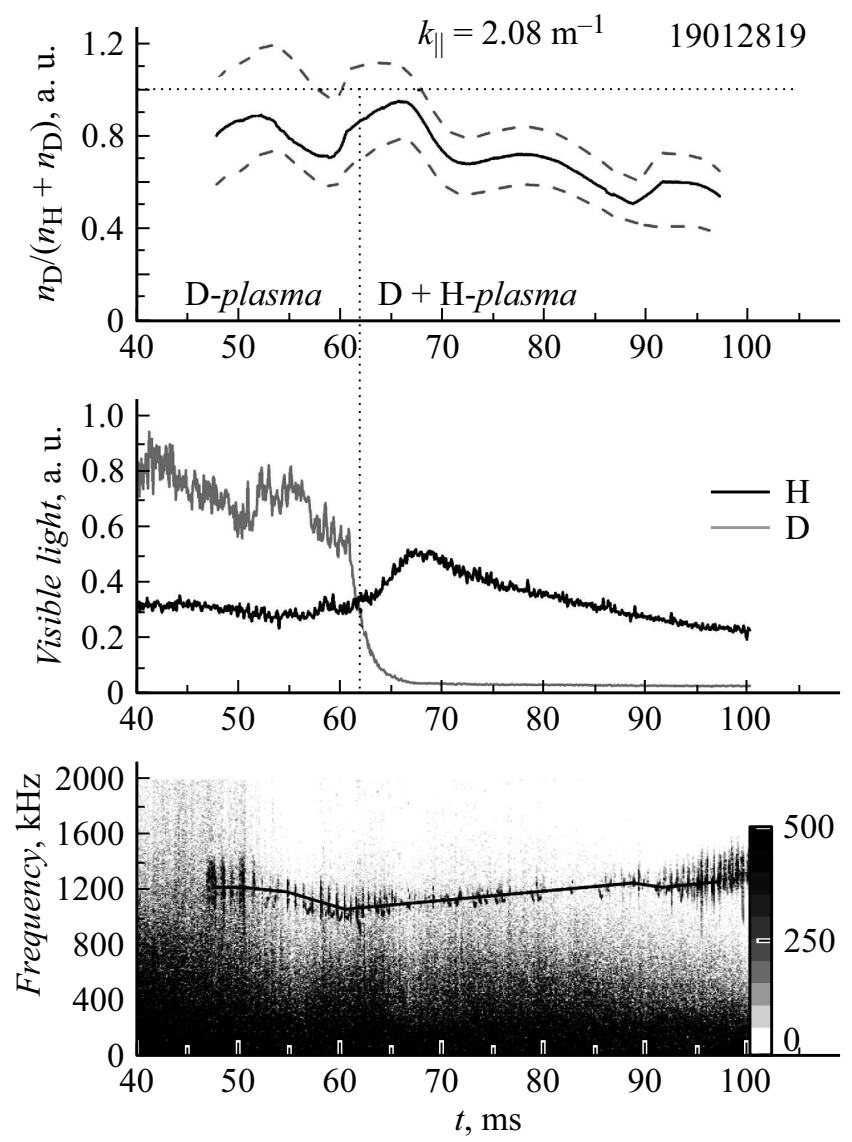

Рис. 2. Относительная концентрация дейтерия, излучение в видимом диапазоне из клапанов напуска газа и спектрограмма сигнала магнитного зонда в разряде 19012819. 

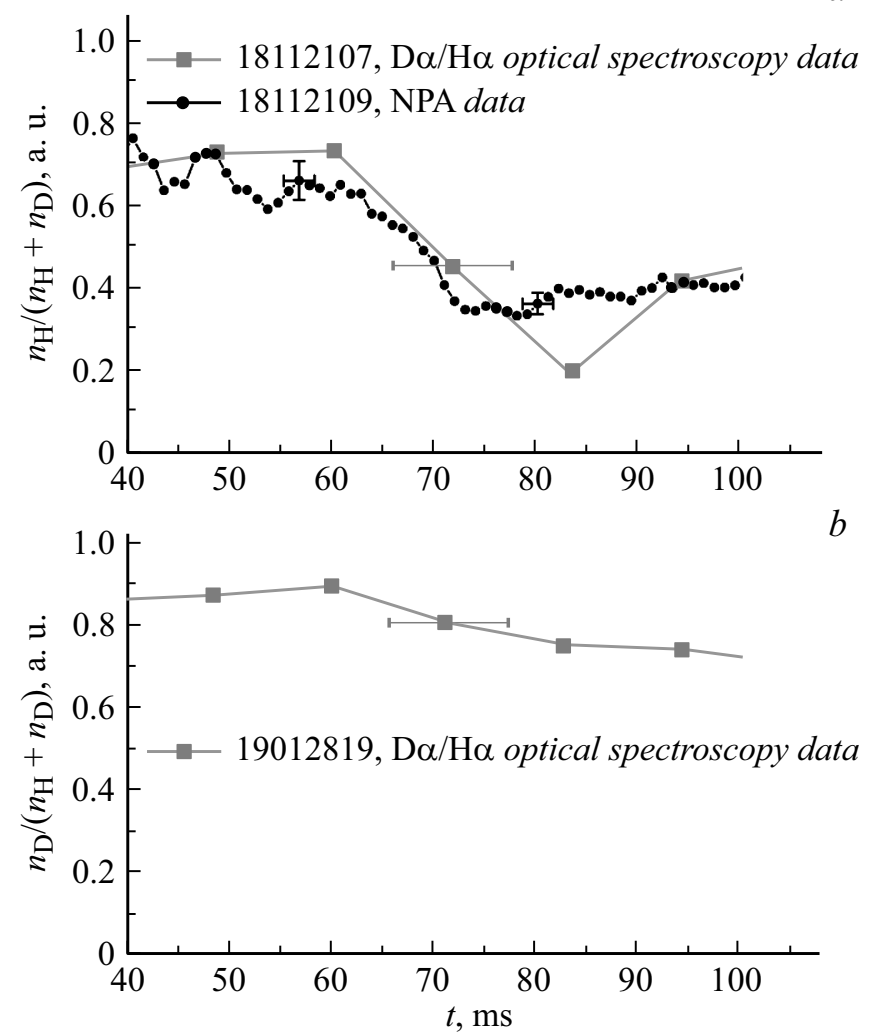

Рис. 3. Относительная концентрация водорода $(a)$ и дейтерия $(b)$, определенная по данным спектроскопических измерений (квадраты) и на основе измерений потоков атомов из плазмы (круги).

водорода происходило через дополнительный клапан, и оно также контролировалось фотодиодом, но в данном случае положение фотодиода было таково, что он регистрировал также и свечение фоновой плазмы. Этим объясняется ненулевой уровень сигнала видимого света из дополнительного клапана в отсутствие напуска газа на рис. 1 и 2, которые иллюстрируют основные результаты нашего исследования. На этих рисунках вертикальная пунктирная линия в момент времени $t=62 \mathrm{~ms}$ обозначает момент начала поступления в камеру токамака „примесного“ газа, который соответствует началу роста сигнала с фотодиода, регистрирующего свечение атомов из дополнительного клапана. В разряде 18112106 (рис. 1) АК начинаются с $50 \mathrm{~ms}$, для создания фоновой плазмы использовался водород, который напускался с 22 по $60 \mathrm{~ms}$. В разряде 19012819 (рис. 2) АК начинаются c $48 \mathrm{~ms}$, для создания фоновой плазмы использовался дейтерий, который напускался с 22 по $60 \mathrm{~ms}$. Момент прекращения поступления „примесного“ газа $t=67 \mathrm{~ms}$ для обоих разрядов определялся по началу спада сигнала фотодиода, установленного вблизи дополнительного клапана.

Результаты применения описанного выше метода для определения изотопного состава плазмы показаны на рис. 1 и 2. Параллельное волновое число выступало в качестве свободного параметра и подбиралось так, чтобы до момента поступления „примесного“ газа изотопное соотношение примерно равнялось единице. Две штриховые линии на графиках эволюции изотопного соотношения показывают ошибку определения этого параметра, обусловленную погрешностью определения частоты АК.

Определенная описанным методом эволюция изотопного соотношения качественно согласуется с результатами спектроскопических измерений (рис. 3). В момент начала поступления „примесного“ газа $t=62 \mathrm{~ms}$ концентрация основного изотопа начинает спадать. В случае напуска водорода в дейтериевую плазму (рис. 3,a) спад продолжается примерно до $85 \mathrm{~ms}$, где сменяется ростом. Аналогичное поведение относительной концентрации наблюдается при определении ее по частоте АК (рис. 1). В случае напуска дейтерия в водородную плазму (рис. $3, b)$ наблюдается монотонный спад концентрации основного изотопа. Аналогичное поведение также можно увидеть на рис. 2.

Как было отмечено выше, АК в токамаке ТУМАН-3М наблюдаются при относительно низких концентрациях $\left(\bar{n}_{e} \leqslant 1.5 \cdot 10^{19} \mathrm{~m}^{-3}\right)$. В таких режимах присутствует высокий уровень жесткого рентгеновского излучения из-за наличия в плазме большого числа убегающих электронов. Это излучение создает некоторые трудности при измерениях потоков атомов перезарядки и определении изотопного состава методом корпускулярной диагностики. При увеличении концентрации $\left(\bar{n}_{e}>1.5 \cdot 10^{19} \mathrm{~m}^{-3}\right)$ уровень жесткого рентгеновского излучения резко снижается, что позволяет использовать корпускулярную диагностику. Можно рассмотреть данные этой диагностики, полученные в разряде 18112109 с помощью анализатора атомов АКОРД-12 [12] (рис. 3, $a$ ). Сценарий напуска рабочих газов в этом разряде был аналогичен сценарию в исследуемых разрядах 18112106 и 19012819 , а его параметры были идентичны рассматриваемым разрядам, за исключением концентрации плазмы, которая в максимуме достигала $\bar{n}_{e}=1.9 \cdot 10^{19} \mathrm{~m}^{-3}$. Как следует из сравнения рис. 1,2 и 3, изотопные соотношения, определенные по частоте АК, по потокам атомов перезарядки и на основе спектроскопических измерений, не противоречат друг другу.

Отметим, что определенное по частоте АК изотопное соотношение соответствует центральной области плазменного шнура, в то время как спектроскопическая диагностика позволяет оценить этот параметр для периферии плазмы, а корпускулярная диагностика дает информацию об изотопном соотношении, усредненном по хорде наблюдения. Поэтому аналогия в поведении изотопного соотношения, определенного тремя разными методами, носит качественный характер.

Рассмотрим возможную причину различного поведения относительных концентраций после $70 \mathrm{~ms}$ (рис. 1 и 2) - в отсутствие внешних источников водорода и дейтерия. После $70 \mathrm{~ms}$ относительная концентрация 
водорода возрастает (рис. 1), а относительная концентрация дейтерия падает (рис. 2). По-видимому, после импульсного напуска дейтерий быстрее теряется из плазмы, чем водород, что означает большее эффективное время удержания частиц для водорода: $\tau_{p_{\mathrm{H}}}^{*}>\tau_{p_{\mathrm{D}}}^{*}$, $\tau_{p}^{*}=\tau_{p} /(1-R)$, где $\tau_{p}-$ время удержания частиц, $R$ - коэффициент рециклинга. Таким образом, различие в эффективном времени удержания, которое можно увидеть из рис. 1 и 2, может быть объяснено различием во времени удержания частиц и/или коэффициенте рециклинга для разных изотопов.

В заключение необходимо отметить, что, хотя предлагаемый метод определения изотопного соотношения по частоте АК нельзя считать прямым (как, например, метод, базирующийся на корпускулярной диагностике плазмы), его несомненным достоинством является простота, а также осуществимость при низких концентрациях плазмы в условиях высокого рентгеновского фонового излучения. В то же время, если сравнивать метод, основанный на анализе АК со спектроскопическими измерениями, его преимуществом, безусловно, является возможность определения изотопного соотношения для центральной области плазмы - области локализации АК.

\section{Финансирование работы}

Исследование альфвеновских колебаний, а также измерения при помощи корпускулярной диагностики проводились при поддержке Российского научного фонда (грант № 16-12-10285-П). Эксперименты на токамаке ТУМАН-3М выполнены при поддержке Физико-технического института им. А.Ф. Иоффе РАН.

\section{Конфликт интересов}

Авторы заявляют, что у них нет конфликта интересов.

\section{Список литературы}

[1] Murari A., Bertalot L., Bonheure G., Conroy S., Ericsson G., Kiptily V., Lawson K., Popovichev S., Tardocchi M., Afanasyiev V., Angelone M., Fasoli A., Kallne J., Mironov M., Mlynar J., Testa D., Zastrow K.D. and JET-EFDA Contributors // Plasma Phys. Control. Fusion. 2005. V. 47. P. B249-B262.

[2] Afanasyev V.I., Mironov M.I., Nesenevich V.G., Petrov M.P., Petrov S.Ya. // Plasma Phys. Control. Fusion. 2013. V. 55. P. 045008 .

[3] Skinner C.H., Stotler D.P., Adler H., Ramsey A.T. // Rev. Sci. Instrum. 1995. V. 66. P. 646-648.

[4] Okada K., Kondo K., Sato S., Nishitani T., Nomura K., Okamoto A., Iwasaki T., Kitajima S., Sasao M. // Rev. Sci. Instrum. 2006. V. 77. P. 10E726.

[5] Hellesen C., Eriksson J., Bindal F., Conroy S., Ericsson G., Hjalmarsson A., Skiba M., Weiszflog $M$ and JET-EFDA Contributors // Nucl. Fusion. 2015. V. 55. 023005.
[6] Stejner M., Korsholm S.B., Nielsen S.K., Salewski M., Bindslev H., Furtula V., Leipold F., Michelsen P.K., Meo F., Moseev D. // Nucl. Fusion. 2012. V. 52. P. 023011.

[7] Watson G.W., Heidbrink W.W., Burrell K.H., Kramer G.J. // Plasma Phys. Control. Fusion. 2004. V. 46. P. 471-487.

[8] Askinazi L.G., Afanasyev V.I., Altukhov A.B., Bakharev N.N., Belokurov A.A., Bulanin V.V., Bykov A.S., Chernyshev F.V., Chugunov I., Dyachenko V.V., Esipov L.A., Gin D., Goncharov P.R., Gurchenko A.D., Gusakov E.Z., Gusev V.K., Heuraux S., Iblyaminova A.D., Irzak M.A., Kantor M.Yu., Kaveeva E.G., Kiviniemi T., Khilkevitch E.M., Khitrov S.A., Khromov N.A., Kornev V.A., Kouprienko D.V., Kurskiev G.S., Lashkul S.I., Lebedev S.V., Leerink S., Melnik A.D., Minaev V.B., Mironov M.I., Miroshnikov I.V., Mukhin E.E., Nesenevich V.G., Niskala P., Novokhatsky A.N., Patrov M.I., Perevalov A.A., Petrov M.P., Petrov A.V., Petrov Yu.V., Popov A.Yu., Rozhansky V.A., Rozhdestvenskiy V.V., Sakharov N.V., Saveliev A.N., Senichenkov I.Yu., Sergeev V.Yu., Shchegolev P.B., Shatalin S.V., Shcherbinin O.N., Shevelev A.E., Sidorov A.V., da Silva F., Smirnov A.I., Stepanov A.Yu., Sysoeva E.V., Teplova N.V., Tolstyakov S.Yu., Tukachinsky A.S., Varfolomeev V.I., Vekshina E.O., Vildjunas M.I., Voronin A.V., Voskoboinikov S.P., Wagner F., Yashin A.Yu., Zhubr N.A. // Nucl. Fusion. 2015. V. 55. P. 104013.

[9] Тукачинский А.С., Аскинази Л.Г., Балаченков И.М., Белокуров А.А., Гин Д.Б., Жубр Н.А., Корнев В.А., Лебедев С.В., Хилькевич Е.М., Чугунов И.Н., Шевелев А.Е. // Письма в ЖТФ. 2016. Т. 42. В. 24. С. 72-78.

[10] Абдуллина Г.И., Аскинази Л.Г., Белокуров А.А., Жубр Н.А., Корнев В.А., Крикунов С.В., Лебедев С.В., Разуменко Д.В., Тукачинский А.С. // Письма в ЖТФ. 2018. Т. 44. В. 3. C. $47-54$.

[11] Testa D., Blanchard P., Panis T. and JET Contributors // Nucl. Fusion. 2015. V. 55. 123010.

[12] Извозчиков А.Б., Петров М.П., Петров С.Я., Чернышев Ф.В., Шустов И.В. // ЖТФ. 1992. Т. 62. В. 2. С. 157163. 